\title{
A Dynamic Emotional Model for Agent Societies
}

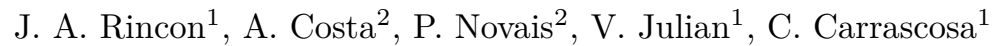 \\ 1 Universitat Politècnica de València. D. Sistemas Informáticos y Computación \\ \{jrincon, vinglada, carrasco@dsic.upv.es\} \\ 2 Centro ALGORITMI, Escola de Engenharia, Universidade do Minho, GuimarÃčes \\ \{acosta, pjon@di.uminho.pt\}
}

\begin{abstract}
This paper presents a first approximation of a dynamic emotional model to be employed in agent societies. The proposed model is based on the $P A D$ emotional model and allows the representation of the emotional contagion phenomena of a heterogeneous group of agents which are capable of express emotions. Moreover, the proposal allows the definition of the social emotion of this group of agents. The model is mainly based on three elements: personality, empathy and affinity. These elements allow the characterization of each individual, causing them susceptible to vary in some degre the emotions of other individuals.
\end{abstract}

\section{Introduction}

What is an emotion? Some psychologists as William James define the emotions as, a representation of physiological change produced by the different stimulus of the environment [1]. Changes in the environment make humans change their emotional states, helping them to make decisions. From a computational perspective, emotions have been employed as a way to improve social simulation processes which require human interactions, but very little work has been done on representing collective emotions and emotion's dinamicity. These states can be affected by an extensive number of internal or external factors. Internal factors are the personality and the cognitive processes such as attention; and external factors are related with the environment, which includes relationships among persons and culture expectations [2]. Over the last few years, different approaches have been proposed trying to model the concept of emotion as well as all of the related elements that affect it. There are already some tested emotional and personality models that can be used to recognize and simulate emotions, such as: OCEAN model [3], OCC emotional models [4], Plutchikś theory [5] and the PAD model [6]. The employment of these models allows the creation of intelligent entities capable of detect and communicate emotions between agents or between humans and agents. In the communication among agents, they can perceive the environment and the emotional state of other agents. In the same sense, if agents interact with humans, agents can perceive the human emotions using different devices such as cameras[7], speech analysis[8], bio-sensors[9], etc..

In most of the existing proposals the emotions are typically modeled as static information which influences the decision of an individual entity without taking 
into account possible interactions with other agents which can give way to an emotion contagion. Emotion contagion may be possible when the model takes into account elements such as situation, affinity, empathy, etc.. Until now, only a few works have tried to model the emotional contagion in computational entities: Saunier and Jones[10] model the emotional contagion suggesting in each agent the separation of the body and the mind; the spiral model by Bosse et al. [11] tries to give a solution to the emotional propagation, distinguishing different factors that influence the emotional contagion.

This paper tries to expand the modelization of emotional contagion, by proposing a dynamic emotional model. The proposed model is based on the $P A D$ emotional model and represents the emotional contagion of a heterogeneous group of entities capable of express and/or communicate emotions. Moreover, it allows to define the social emotion of a group of agents. To define the model we employ concepts like empathy, affinity and personality of each entity.

The employed concepts open the door to the construction of scenarios highly related to real-life events. Projects like the iGenda $[12,13]$ require virtual actors that have responses similar to human responses.

\section{Related Work}

The emotional states are defined as the way to express emotion by humans being in a period of time. These emotion are not static and can be propagated through the environment, begin widely used in crowd simulation. It is essential to these applications too have the ability of emulating emotion as they are used to the decision making process. In crowd simulation the most common emotional state is fear, which allows the creation of emergency evacuation simulations [14], [15]. Nevertheless, these simulations try to predict the behavior of humans in distress. These simulations have helped to design buildings, evacuation routes and simulate how the police, firefighters and ambulance may optimally respond to a disaster situation [16]. However human being have a whole range of emotions that can be propagated to other agents, such as: happiness, sadness, anger among others. To propagate these emotions the Newtonian Emotion System (NES) [17] is designed for multi-agent system, establishing the three laws of motion presented by Newton. In the Newton dynamic the aim is study of movements of objects and the origin of these movements, where each object is represented by a particles system. Each one of these particles have internal properties which makes them different to the other particles properties as the mass, length, with and height, among others this provide to the object a different behavior when external forces are acting on it. The application of these forces on a particle can changes your direction and velocity or knows if this particle is attracted to another. The authors based on their model in the Newton laws and apply some of the concepts presented by Newton, concepts as force, mass, acceleration and velocity. Using this concepts the author defined two laws of emotion dynamics, this two law is based on the laws of dynamic of Newton. 
Other works have tried to introduce the contagion effect that humans can feel in multiple situations. One of these works is the emotional contagion spiral model [18]. This model tries to give a solution to the emotional propagation, distinguishing among different factors that influence in the emotional contagion. This model is based on a emotional model that was proposed by Barsade [19], which includes six hypotheses about how is produced the propagation of emotions. This work is applied in an evacuation simulation scenario, taking into account how human behaviors are affected by the dynamicity and propagation of emotions. Nevertheless, the complexity of these analysis provoques that these approaches are limited to only one emotion, in this case fear. So, behaviors of simulated agents are also affected by only one emotion.

This work tries to pose a possible solution to this problem, pretending to give a first approximation of a dynamic emotional model which allows the representation of the emotional contagion of a heterogeneous group of entities capable of express and/or communicate emotions. In this sense, nexte section explains in detail the proposed model.

\section{Dynamic Emotional Model}

This section proposes a dynamic emotional model based on the PAD emotional model. This model will represent the emotional contagion of a heterogeneous group of entities capable of expressing and/or communicating emotions.

Before defining the dynamic emotional model, it is necessary to define the representation of an emotional state of an agent on the PAD model. The emotion of an agent $a g_{i}$ in an instant $t\left(\vec{E}_{t}\left(a g_{i}\right)\right)$ is defined as a vector in $\mathbb{R}^{3}$, represented by the components that make up the $P A D$ emotional model. The variation of each component allows to modify the emotional state of the agent (Equation 1).

$$
\vec{E}_{t}\left(a g_{i}\right)=\left[P_{t}\left(a g_{i}\right), A_{t}\left(a g_{i}\right), D_{t}\left(a g_{i}\right)\right]
$$

This representation in $\mathbb{R}^{3}$ allows us to see emotions as a system of particles. They attract or repel depending on the internal properties of each one of them. These particles have the ability to move around the space because these particles have internal properties as Mass. The mass in a particle is a measure of the amount of matter that has a body, and one of the properties related to it is that it is proportional to the resistance to be attracted by others.

The attraction carried out in the $P A D$ space reflects the emotional contagion between entities. An entity will be more easily suffer from contagion of other emotions according to different factors. The main factor, depending on the own entity is called Empathy. The empathy is a psychological motivator for helping others in distress [20]. The empathy could be defined as the ability to feel what other people feel. The empathy denotes a deep emotional understanding of another's feelings or problems, while sympathy is more general and can apply to small annoyances or setbacks. Our dynamical model uses this psychological concept, allowing agents to have an empathy level. The Empathy Level of an agent $a g_{i}$, denoted $\varepsilon\left(a g_{i}\right)$, represents a value in the range $[0,1]$ indicating the 
ability of agent $a g_{i}$ to perceive what another agent may feel. In the $P A D$ space, the mass of agent $a g_{i}\left(m\left(a g_{i}\right)\right)$ is defined as the inverse of empathy (Equation 2) as an indicator of the difficult to be attracted by others, that is to be contagied by other emotions as $m\left(a g_{i}\right)$ increases.

$$
m\left(a g_{i}\right)=\frac{1}{\left|\varepsilon\left(a g_{i}\right)\right|}
$$

Another important factor in the emotional contagion is the relationship between the emotion source and the emotion receiver, that is, the Affinity existing between them. It is not the same to perceive the emotions of a close acquaintance than a stranger. The Affinity Level between two agents $a g_{i}$ and $a g_{j}$ at instant $t\left(A f_{t}\left(a g_{i}, a g_{j}\right)\right)$ is a value between $[-1,1]$ that describes the level of affinity between agents $a g_{i}$ and $a g_{j}$, being -1 the value dedicated to sworn enemies, 0 to perfect strangers and 1 to best of friends. The last factor to take into account in the emotion dynamics is the physical distance between the emotion source and the emotion receiver $\left(D_{t}\left(a g_{i}, a g_{j}\right)\right.$ to denote the physical distance between entities $a g_{i}$ and $a g_{j}$ at instant $\left.t\right)$.

The emotional dynamics described is based on the Newton universal attraction law. Newton's law of universal gravitation states that any two bodies in the Universe attract each other with a force that is directly proportional to the product of their masses and inversely proportional to the square of the distance between them. Based on this theory, we define the force that an agent $a g_{j}$ makes over an agent $a g_{i}$ at instant $t\left(\vec{F}_{t}\left(a g_{i}, a g_{j}\right)\right)$ to attract or repulse it in the $P A D$ space, that is, this force will control the emotion contagion between all the agents. The emotional force is a vector in $\mathbb{R}^{3}$ space. This vector measures the emotional change in the $P A D$ space (Equation 3 ).

$$
\vec{F}_{t}\left(a g_{i}, a g_{j}\right)=\frac{\varepsilon\left(a g_{i}\right) \cdot A f_{t}\left(a g_{i}, a g_{j}\right)}{2^{D_{t}\left(a g_{i}, a g_{j}\right)}} \cdot\left\|\vec{E}_{t}\left(a g_{i}\right)-\vec{E}_{t}\left(a g_{j}\right)\right\|
$$

$\vec{F}_{t}\left(a g_{i}, a g_{j}\right)$ represents the force vector, which help us to know if the emotion of the agent $a g_{i}$ is attracted by the agent $a g_{j} . \varepsilon\left(a g_{i}\right)$ represents the emphatic level of entity $a g_{i}$, and $A f_{t}\left(a g_{i}, a g_{j}\right)$ represents the affinity level between $a g_{i}$ and $a g_{j}$ at instant $t . D_{t}\left(a g_{i}, a g_{j}\right)$ is the physical distance between $a g_{i}$ and $a g_{j}$ at instant $t$ and $\vec{E}_{t}\left(a g_{i}\right)$ represents the emotion of the $a g_{i}$ at instant $t$ and $\vec{E}_{t}\left(a g_{j}\right)$ represents the emotion of the $a g_{j}$ at instant $t$. According to this, we define the Emotional Attraction Force of agent $a g_{i}$ at instant $t\left(\overrightarrow{E A F}_{t}\left(a g_{i}\right)\right)$ as the combination of all the attraction forces over agent $a g_{i}$ at instant $t$ (Equation 4 ).

$$
\overrightarrow{E A F}_{t}\left(a g_{i}\right)=\sum_{\forall a g_{j} \neq a g_{i}} \vec{F}_{t}\left(a g_{i}, a g_{j}\right)
$$

To calculate the new emotion of agent $a g_{i}$ at instant $t+1$ and assuming that there is no external stimuli that may change agent $a g_{i}$ emotion out of the rest of entities in the system, it will be calculated according to movement in the $P A D$ space. To get this new emotion it is necessary to use the second law of Newton's or the fundamental principle of dynamics. Based on this law, the $\overrightarrow{E A F}_{t}\left(a g_{i}\right)$ is 
used to calculate the emotional acceleration of agent $a g_{i}$ at instant $t\left(\vec{a}_{t}\left(a g_{i}\right)\right)$. This acceleration is the emotional variation per time unit of agent $a g_{i}$ emotion (Equation 5).

$$
\overrightarrow{E A F}_{t}\left(a g_{i}\right)=m\left(a g_{i}\right) \cdot \vec{a}_{t}\left(a g_{i}\right)
$$

Once the emotional acceleration $\vec{a}_{t}\left(a g_{i}\right)$ is calculated, the emotional velocity of entity $a g_{i}$ at instant $t$ can be obtained $\left(\vec{v}_{t}\left(a g_{i}\right)\right)$. This is a measure of the emotional propagation velocity within the $P A D$ space (Equation 6).

$$
\vec{v}_{t}\left(a g_{i}\right)=\vec{a}_{0}\left(a g_{i}\right)+\left(\vec{a}_{t}\left(a g_{i}\right) \cdot t\right)
$$

Finally, it is necessary to calculate the new $P A D$ emotion for entity $a g_{i}$ at instant $t+1\left(\vec{E}_{t+1}\left(a g_{j}\right)\right)$ (Equation 7$)$.

$$
\vec{E}_{t+1}\left(a g_{j}\right)=\vec{E}_{t}\left(a g_{j}\right)+\left(\vec{v}_{t}\left(a g_{i}\right) \cdot t\right)
$$

It is important to consider that emotions within the $P A D$ space do not present any opposition by the environment, e.g., there is no friction causing a reduction of speed. There is no inercia affecting the emotions within the PAD space thus, there are no oscillations. This swing up was eliminated by adding this restriction to the model if $\overrightarrow{E A F}_{t}\left(a g_{i}\right)=0$ then $\vec{v}_{t}\left(a g_{i}\right)=0$.

The proposed dynamic model allows us to model and represent the emotional contagion phenomena among different intelligent agents. Nevertheless, these entities typically are not alone in the environment but are part of a group of agents. Our proposal is to model not only how an agent is influenced by other agents but also how the group of agents as a whole can be emotionally affected by its components. To do this, we need to define a social emotional model, which allows to calculate and represent the social emotion of a group of intelligent entities. Next subsection presents the proposed model for representing social emotions.

\subsection{Social Emotional Model}

The aim of the social emotional model is to obtain the social emotion of a group of heterogeneous agents in an specific instant. This model is composed by a triplet that allows us to define the social emotion $(S E)$ [21] for a group of $\mathrm{n}$ agents $A g=\left\{a g_{1}, a g_{2}, \ldots, a g_{n}\right\}$ at instant $t$ (Equation 8 ).

$$
S E_{t}(A g)=\left(\overrightarrow{C E}_{t}(A g), \vec{m}_{t}(A g), \vec{\sigma}_{t}(A g)\right)
$$

Where $\overrightarrow{C E}_{t}(A g)$ is a vector in the PAD space, where each one of its components is calculated averaging the $P, A$, and $D$ values, respectively of the $\mathrm{n}$ agents forming the set $A g$ (Equation 9). These averages will enable us to determine where the central emotion $(C E)$ of this group of agents is and to visualize it in the $P A D$ space.

$$
\begin{aligned}
\bar{P}_{t}(A g) & =\frac{\sum_{i=1}^{n} P_{t}\left(a g_{i}\right)}{n}, \bar{A}_{t}(A g)=\frac{\sum_{i=1}^{n} A_{t}\left(a g_{i}\right)}{n}, \bar{D}_{t}(A g)=\frac{\sum_{i=1}^{n} D_{t}\left(a g_{i}\right)}{n}, \\
\overrightarrow{C E}_{t}(A g) & =\left[\bar{P}_{t}(A g), \bar{A}_{t}(A g), \bar{D}_{t}(A g)\right]
\end{aligned}
$$


The $\vec{m}_{t}(A g)$ can indicate if there exist agents having their emotional state far away from the central emotion. The Euclidean distance is used to calculate the maximum distances between the emotion of each agent respect to the $\overrightarrow{C E}$ (Equation 10, 11, 12, 13) as follows.

$$
\begin{gathered}
m P_{t}(A g)=\max \left(\sqrt{\left(P_{t}\left(a g_{i}\right)-\bar{P}_{t}(A g)\right)^{2}}\right), \forall a g_{i} \in A g \\
m A_{t}(A g)=\max \left(\sqrt{\left(A_{t}\left(a g_{i}\right)-\bar{A}_{t}(A g)\right)^{2}}\right), \forall a g_{i} \in A g \\
m D_{t}(A g)=\max \left(\sqrt{\left(D_{t}\left(a g_{i}\right)-\bar{D}_{t}(A g)\right)^{2}}\right), \forall a g_{i} \in A g \\
\vec{m}_{t}(A g)=\left[m P_{t}(A g), m A_{t}(A g), m D_{t}(A g)\right]
\end{gathered}
$$

The $\vec{\sigma}(A g)$ or standard deviation (SD) allows the calculation of the level of emotional dispersion of this group of agents around the central emotion $\overrightarrow{C E}(\mathrm{Ag})$ for each component of the PAD(Equation 14).

$$
\begin{aligned}
\sigma P_{t}(A g) & =\sqrt{\frac{\sum_{i=1}^{n}\left(P_{t}\left(a g_{i}\right)-\bar{P}_{t}(A g)\right)^{2}}{n}}, \forall a g_{i} \in A g \\
\sigma A_{t}(A g) & =\sqrt{\frac{\sum_{i=1}^{n}\left(A_{t}\left(a g_{i}\right)-\bar{A}_{t}(A g)\right)^{2}}{n}}, \forall a g_{i} \in A g \\
\sigma D_{t}(A g) & =\sqrt{\frac{\sum_{i=1}^{n}\left(D_{t}\left(a g_{i}\right)-\bar{D}_{t}(A g)\right)^{2}}{n}}, \forall a g_{i} \in A g
\end{aligned}
$$

The result of each of the above equations can be represented as a vector (Equation 15), which allow to determine the level of emotional dispersion.

$$
\vec{\sigma}_{t}(A g)=\left[\sigma P_{t}(A g), \sigma A_{t}(A g), \sigma D_{t}(A g)\right]
$$

From this definition, it can be deduced that:

1. if $\vec{\sigma}_{t}(A g)>>[\mathbf{0 , 0 , 0}$, the group has a high emotional dispersion, i.e. the members of the group have different emotional states.

2. if $\vec{\sigma}_{t}(A g) \cong[\mathbf{0 , 0}, \mathbf{0}]$, the group has a low emotional dispersion, this means that individuals have similar emotional states.

This model takes into account that at some stage you may have two or more agent groups and each group has its own social emotion or have a single group which wants to move to a target emotion. This will allow to measure the emotional distance between the current social emotional group and a possible emotional target. This approach can be used as a feedback in the decision making process in order to take actions to try to move the social emotion to a particular 
area of the $P A D$ space or to allow that the emotional state of a group of agents can be approached or moved away from other groups of agents (Equation 16).

$$
\Delta_{S E}: S E_{t}\left(A g^{i}\right), S E_{t^{\prime}}\left(A g^{j}\right) \rightarrow[0,1]
$$

According to this profile, Equation 17 shows how we calculate this emotional variation. The equation calculates three distances corresponding to the three components of the $S E$.

$$
\begin{array}{r}
\Delta_{S E}\left(S E_{t}\left(A g^{i}\right), S E_{t^{\prime}}\left(A g^{j}\right)\right)=\frac{1}{2}\left(\omega_{c} \Delta\left(\overrightarrow{C E}_{t}\left(A g^{i}\right), \overrightarrow{C E}_{t^{\prime}}\left(A g^{j}\right)\right)\right. \\
\left.+\omega_{d} \Delta\left(\vec{m}_{t}\left(A g^{i}\right), \vec{m}_{t^{\prime}}\left(A g^{j}\right)\right)+\omega_{v} \Delta\left(\vec{\sigma}_{t}\left(A g^{i}\right), \vec{\sigma}_{t^{\prime}}\left(A g^{j}\right)\right)\right) \\
\text { where } \omega_{c}+\omega_{d}+\omega_{v}=1 ; \quad \omega_{c}, \omega_{d}, \omega_{v} \in[0,1]
\end{array}
$$

and $\Delta$ calculates the distance between two vectors. As every dimension of the $P A D$ space is bounded between $[-1,1]$, each $\Delta$ will give values between $[0,2]$. Therefore, $\Delta_{S E}$ will have a range between $[0,1]$. Calculating the distance among social emotions allows the study of the behaviour of emotional-based agents, either minimizing or maximizing the $\Delta_{S E}\left(S E_{t}\left(A g^{i}\right), S E_{t^{\prime}}\left(A g^{j}\right)\right)$ function. This way, it can be extrapolated the knowledge about if an agent group approaches or moves away from a specific emotional state. To achieve this, it is necessary to modify through stimuli the individual emotions of each agent and therefore changing the social emotion.

Using this model is possible to determine the emotional distance among different groups of agents or between the same group in different instants of time. This will allow us to measure the emotional distance between the current social emotional group and a possible emotional target. Moreover, the combination of the presented models allows us to model and represent the emotional contagion of a heterogeneous group of agents and observe how it influences the social emotion of that group of agents.

\section{Case Study}

Different tests have been done in order to validate the proposed model. Concretely, a simulation prototype was implemented in Python (using a jupyter ${ }^{3}$ notebook with numpy and matplotlib libraries). The simulation experiments were conducted to evaluate different aspects and to try to show the correct behavior of the proposed model. Visualization of results has been done using three different kind of images:

- PAD space representation: a 3D representation of the emotional states in the PAD space. In each graphic current emotional states of each agent and the social emotion of the existing groups are represented.

\footnotetext{
${ }^{3}$ http://jupyter.org
} 
- Physical space position representation: a 2D representation of the different agents, similar to a graph where each agent is a node situated in its physical coordinates $(\mathrm{x}, \mathrm{y})$. The size of the agent is inversely proportional to its empathy and if there is any affinity between agents, it will be represented by a link joining them. Finally, a sequence of colors (see Figure 1) is defined as a way for representing the current emotion of each agent.

- Social emotional evolution: a 2D representation of the evolution of the different values composing the Social Emotion $\left(S E_{t}(A g)\right)$ :

- $\overrightarrow{C E}_{t}(A g)=\left[\bar{P}_{t}(A g), \bar{A}_{t}(A g), \bar{D}_{t}(A g)\right]$, represented in the figure as $C E P$, $C E A$ and $C E D$, respectively.

- $\vec{m}_{t}(A g)=\left[m P_{t}(A g), m A_{t}(A g), m D_{t}(A g)\right]$, represented in the figure as maxDistP, maxDistA and maxDistD, respectively.

- $\vec{\sigma}_{t}(A g)=\left[\sigma P_{t}(A g), \sigma A_{t}(A g), \sigma D_{t}(A g)\right]$, represented in the figure as $\operatorname{stdP}$, stdA and $s t d D$, respectively.

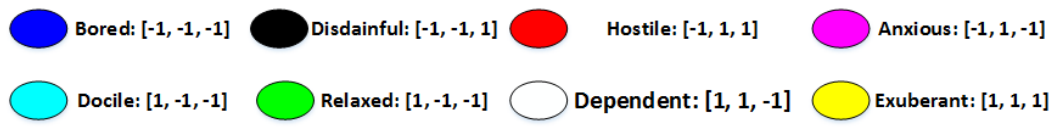

Fig. 1: Color representation for the different emotions

The experiments have been grouped into three situations changing the characteristics of the agents' groups. Moreover, each experiment includes different cases changing the affinity and empathy levels of the agents and also the physical distance among agents. The different proposed experiments are listed in Table 1

\begin{tabular}{|c|c|c|c|c|c|c|}
\hline \multirow{2}{*}{ Experiment } & \multirow{2}{*}{\multicolumn{2}{|c|}{ \# agents }} & \multirow{2}{*}{ Empathy } & \multirow{2}{*}{ Affinity } & \multicolumn{2}{|c|}{ Physical distance } \\
\hline & & & & & Case 1 & Case 2 \\
\hline $1 \mathrm{st}$ & 1 group of 10 agents & $\begin{array}{l}\text { a) } \\
\text { b) } \\
\text { c) } \\
\text { d) }\end{array}$ & $\begin{array}{l}0 \\
0 \\
1 \\
1\end{array}$ & $\begin{array}{l}0 \\
1 \\
0 \\
1\end{array}$ & $\begin{array}{c}\text { All agents } \\
\text { have distance } \\
0\end{array}$ & $\begin{array}{l}\text { All agents have } \\
\text { random distances } \\
\text { between } 0 \text { and } 20\end{array}$ \\
\hline 2nd & $\begin{array}{c}1 \text { group of } 10 \text { agents } \\
\text { (one agent with Empathy } \\
\text { and Affinity }=0 \text { ) }\end{array}$ & $\begin{array}{l}\text { a) } \\
\text { b) } \\
\text { c) } \\
\text { d) }\end{array}$ & $\begin{array}{l}0 \\
0 \\
1 \\
1\end{array}$ & $\begin{array}{l}0 \\
1 \\
0 \\
1\end{array}$ & $\begin{array}{c}\text { All agents } \\
\text { have distance } \\
0\end{array}$ & $\begin{array}{l}\text { All agents have } \\
\text { random distances } \\
\text { between } 0 \text { and } 20\end{array}$ \\
\hline $3 r d$ & $\begin{array}{c}1 \text { group of } 5 \text { agents } \\
\text { and } 1 \text { group of } 10 \text { agents }\end{array}$ & $\begin{array}{l}\text { a) } \\
\text { b) } \\
\text { c) } \\
\text { d) }\end{array}$ & $\begin{array}{l}0 \\
0 \\
1 \\
1\end{array}$ & $\begin{array}{l}0 \\
1 \\
0 \\
1\end{array}$ & $\begin{array}{c}\text { All agents } \\
\text { have distance } \\
0\end{array}$ & $\begin{array}{l}\text { All agents have } \\
\text { random distances } \\
\text { between } 0 \text { and } 20\end{array}$ \\
\hline
\end{tabular}

Table 1: Summary of proposed experiments 


\subsection{First Experiment}

The first experiment tried to evaluate how a group of heterogeneous agents evolve in the emotional space according to the dynamic model. To do this, we implemented a set of 10 agents with a randomized initial emotional state. In order to evaluate the emotional behavior in the agent group, different situations have been defined changing the empathy and affinity values of each agent. Moreover the physical distance has also changed from a minimum distance of 0 meters up to a maximum distance of 20 meters. For reasons of brevity only two of the combinations are described.

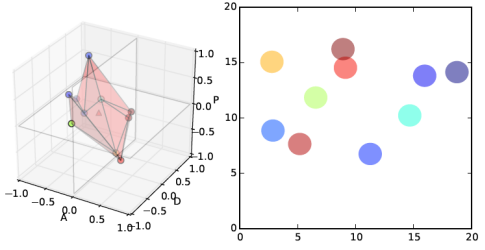

(a) Initial

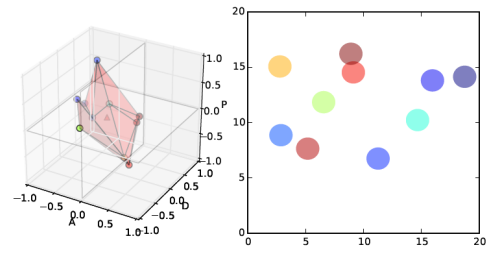

(b) Final

Fig. 2: One group of 10 agents (with Empathy=0, Affinity=0 and distance between agents $>0$ )

First one is the corresponding to all the empathies and affinities between agents to 0 , that is, a set of agents that has not any relationship between them and that are not moved by the emotions they feel around them. In this situation, the model works as expected, as the agents do not change their emotions. Figure 2 shows one execution of this first situation of this example by a PAD space representation and a Physical space position representation for the initial and final stages of the execution.

Alternatively, Figure 3 represents a situation where agents have a maximum value of the empathy and affinity levels. As we can see, the initial stage for the PAD values of the agents is the same of the previous situation (as can be observed in the corresponding PAD space representation). As this situation has different affinities and empathies, there exist links connecting agents in the Physical space position representation. This situation represents a group of agents that can be considered good friends and very sensitive to their friends emotions. As they are close enough (in a range of $[0,20]$ meters), their emotions are contaged tending to collapse in the PAD space (as is observed in the Figure $4 \mathrm{~b}$ - left). This evolution can be observed, at individual level, in the evolution of the PAD space representation, and in the evolution of the colors of the agents in the PAD space representation and in the Physical space position representation. On the other hand, Figure 4c shows how fast is the convergence of the social emotional values 


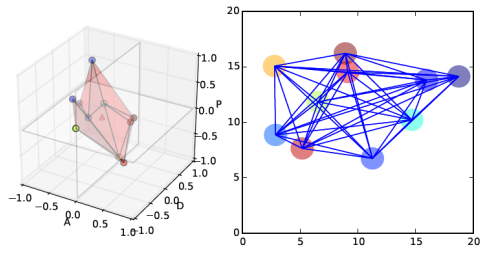

(a) Initial

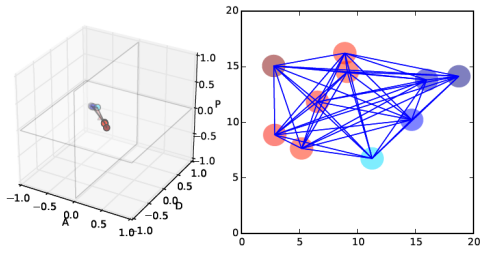

(b) Final

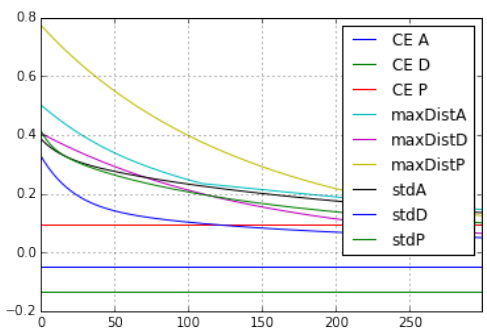

(c) Social emotional evolution

Fig. 3: One group of 10 agents (with Empathy=1, Affinity=1 and all the agents with distance $>0$ )

during the experiment. The relevance of these experiments is the validation that all the situations have the expected behavior according to the proposed model.

\section{$4.2 \quad$ Second Experiment}

The second experiment is trying to observe how the emotional state of the group is disturbed by an odd agent without empathy and affinity with any agent. Scenarios proposed in this experiment are affected in the emotional states of the group due to the emotional response generated by the odd agent. As an example we can see the scenario proposed in Figure 5a where all the agents of the group have the maximum value of the empathy and affinity levels except the odd agent (an initial situation similar to the one used in the Figure 4a). As we can see, the final situation shows a non perfect grouping of all the agents due to the distorsion caused by the odd agent. This can be observed too in the temporal evolution of the social emotional values, if compared with Figure 3.

\subsection{Third Experiment}

Finally, the third experiment was centered in analyzing how two disimilar groups of agents change their emotional states following the proposed model.

Figure 5 represents a scenario where there exists one group of ten agents and another group of five agents with the maximum level of empathy and affinity 


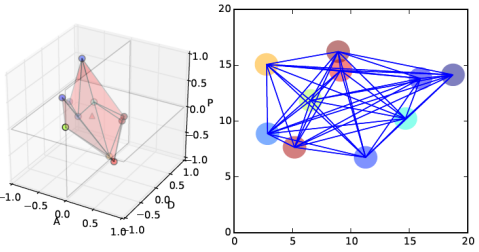

(a) Initial

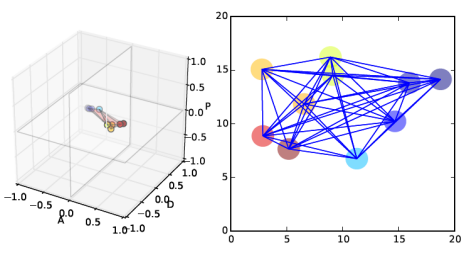

(b) Final

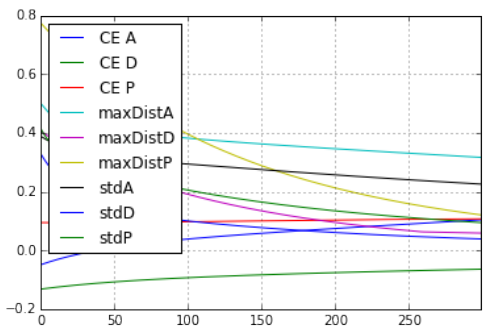

(c) Social emotional evolution

Fig. 4: One group of 10 agents with an odd agent (with Empathy=1, Affinity=1 and all the agents with distance $>0$ )

inside the group and the minimum distance between them. In this case, agents of each group are close to each other as can be expected. Regarding the temporal evolution, it is more evident in the case of the smallest group, where the social emotional parameters are more homogeneous than in the largest group.

\section{Conclusions and future work}

A new model for representing the emotional contagion has been presented in this paper. The goal of this model is to give a first approach for traction and emotional contagion in a group of intelligent entities. In this model the emotions of each agent are represented using PAD emotional model, which allows the representation of individual emotions in intelligent entities. The proposed model of dynamic emotion uses the individual emotions of each entity of a group, to obtain the level of emotional attraction between them. This model use the dynamic Newton Law and universal gravitation law, to calculate the attraction level $\left(\overrightarrow{E A F}_{t}\left(a g_{i}\right)\right)$ and the new emotion of each agent $\left(\vec{E}_{t+1}\left(a g_{j}\right)=\vec{E}_{t}\left(a g_{j}\right)+\left(\vec{v}_{t}\left(a g_{i}\right) \cdot t\right)\right)$. These definitions allow to calculate the emotional attraction between entities or groups. Moreover, it is possible to obtain the resulting emotion of the attraction in a $(t+1)$, as well as the emotional propagation velocity $\left(\vec{v}_{t}\left(a g_{i}\right)\right)$. Considering these elements is possibly to know how is the emotional distribution among the agent group and to use this information to take on a decision and change your behaviour. 


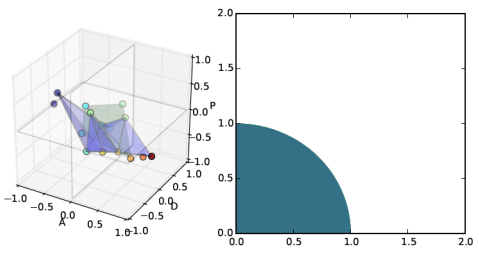

(a) Initial

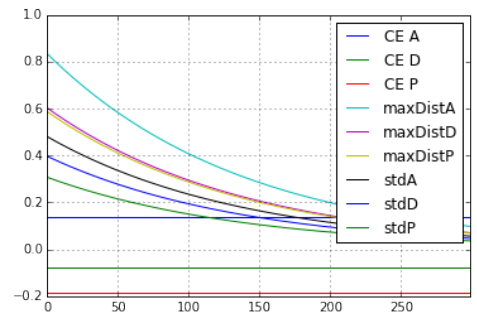

(c) Social emotional evolution of $5(\mathrm{~d})$ Social emotional evolution of agents

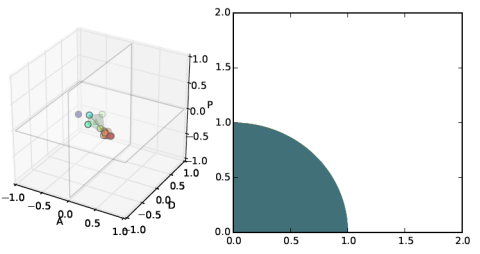

(b) Final

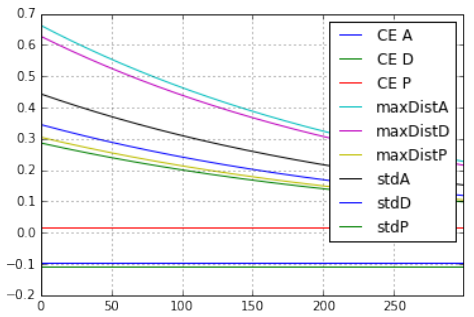

10 agents

Fig. 5: One group of 10 agents and another group of 5 agents (with Empathy=1, Affinity $=1$ and all the agents with distance $=0$ )

As future work, we want to introduce emotion recognition using face and physiological signals, using this information to allow us to obtain the human emotion and to simulate emotional contagion between humans and agents.

\section{References}

1. W. James. What Is an Emotion? Wilder Publications, 2007.

2. N A. Fox and S D. Calkins. The development of self-control of emotion: Intrinsic and extrinsic influences. Motivation and emotion, 27(1):7-26, 2003.

3. R R. McCrae and O P. John. An introduction to the five-factor model and its applications. Journal of personality, 60(2):175-215, 1992.

4. Andrew Ortony. The Cognitive Structure of Emotions. Cambridge University Press, May 1990.

5. H. Kellerman and R. Plutchik. Emotion: Theory, Research and Experience. Vol.1, , Theories of Emotion. Number v. 1. Academic Press, 1980.

6. A Mehrabian. Analysis of affiliation-related traits in terms of the PAD temperament model. The Journal of Psychology, 131(1):101-117, January 1997.

7. C Busso, Z Deng, and et al. Analysis of emotion recognition using facial expressions, speech and multimodal information. In Proceedings of the 6th International Conference on Multimodal Interfaces, ICMI '04, pages 205-211, New York, NY, USA, 2004. ACM.

8. H. K. Palo, M Narayana Mohanty, and M Chandra. Use of Different Features for Emotion Recognition Using MLP Network. In Computational Vision and Robotics, number 332, pages 7-15. Springer, 2015. 
9. D Oude Bos. EEG-based emotion recognition. The Influence of Visual and Auditory Stimuli, pages 1-17, 2006.

10. Julien Saunier and Hazaël Jones. In Proc. of the 2014 international conference on Autonomous agents and multi-agent systems, pages 645-652.

11. Tibor Bosse, Rob Duell, Zulfiqar A. Memon, Jan Treur, and C. Natalie Van Der Wal. A multi-agent model for emotion contagion spirals integrated within a supporting ambient agent model. In Principles of Practice in Multi-Agent Systems, pages 48-67. Springer, 2009.

12. Ângelo Costa, José Carlos Castillo, Paulo Novais, Antonio Fernández-Caballero, and Ricardo Simoes. Sensor-driven agenda for intelligent home care of the elderly. Expert Systems with Applications, 39(15):12192-12204, nov 2012.

13. Angelo Costa, Paulo Novais, and Ricardo Simoes. A caregiver support platform within the scope of an ambient assisted living ecosystem. Sensors, 14(3):5654-5676, jan 2014.

14. Carole Adam, Richard Canal, Benoit Gaudou, Ho Tuong Vinh, Patrick Taillandier, and others. Simulation of the Emotion Dynamics in a Group of Agents in an Evacuation Situation. In Principles and Practice of Multi-Agent Systems, pages 604-619. Springer, 2012.

15. Soumya Banarjee, Crina Grosan, and Ajith Abraham. Emotional ant based modeling of crowd dynamics. In Symbolic and Numeric Algorithms for Scientific Computing, 2005. SYNASC, pages 8-pp. IEEE, 2005.

16. Glenn I. Hawe, Graham Coates, Duncan T. Wilson, and Roger S. Crouch. Agentbased simulation for large-scale emergency response: A survey of usage and implementation. ACM Computing Surveys (CSUR), 45(1):8, 2012.

17. Valentin Lungu. Newtonian emotion system. In Intelligent Distributed Computing VI, pages 307-315. Springer, 2013.

18. Tibor Bosse, Mark Hoogendoorn, and et al. Modelling collective decision making in groups and crowds: Integrating social contagion and interacting emotions, beliefs and intentions. JAAMAS, 27(1):52-84, July 2013.

19. Sigal G. Barsade. The ripple effect: Emotional contagion and its influence on group behavior. Administrative Science Quarterly, 47(4):644-675, December 2002.

20. Nicole M. McDonald, Daniel S. Messinger, A. Acerbi, J. A. Lombo, and J. J. Sanguineti. The development of empathy: How, when, and why. Moral Behavior and Free Will: A Neurobiological and Philosophical Aprroach, pages 341-368, 2011.

21. J. A. Rincon, V. Julian, and C. Carrascosa. Social Emotional Model. In Advances in Practical Applications of Agents, Multi-Agent Systems, and Sustainability: The PAAMS Collection, pages 199-210. Springer, 2015. 